\title{
INVESTIGACIONES
}

\section{Diferencias en la elección de establecimiento educacional para la población local e inmigrantes: Caso chileno}

\author{
School choice differences between the local and immigrant population: Chilean case
}

\author{
Juan Pedro Eberhard Aguirre ${ }^{a}$, Catalina María Lauer Zegarra ${ }^{b}$ \\ ${ }^{a}$ Universidad Adolfo Ibáñez, Chile. \\ juan.eberhard@uai.cl \\ ${ }^{b}$ Universidad Adolfo Ibáñez, Chile. \\ catalinalauer@hotmail.com
}

\section{RESUMEN}

La inmigración es un fenómeno en aumento en Chile en los últimos años. En particular, más de la mitad de los inmigrantes que llega al país lo hace con sus hijos, quienes ingresan al sistema educativo. En este estudio buscamos entender qué tipo de establecimientos eligen las familias chilenas e inmigrantes y en caso de encontrar diferencias estudiar en qué radican. Utilizamos bases de datos del Ministerio de Educación para estimar un modelo probit de school choice ampliamente utilizado en la literatura de elección escolar. Estimamos el modelo por separado para inmigrantes y chilenos para comparar las posibles diferencias en las variables que afectan la decisión de establecimiento. Encontramos que, si bien inmigrantes y chilenos son similares y sus decisiones también lo son, valoran ciertas características de diversa manera y que la mayor diferencia en elección se debe a su composición sociodemográfica.

Palabras Clave: Sistema de voucher, inmigrantes, educación.

\begin{abstract}
Immigration is an increasing phenomenon in Chile in recent years. In particular, more than half of the immigrants that arrive to the country do so with their children, who enroll into the educational system, In this paper, we seek to understand which type of educational institution chilean and immigrant families choose and, in case of finding differences, study them. We use education ministry's databases to estimate a probit model of school choice widely used in the literature of school choice. We separately estimate the model for immigrants and chileans in order to compare the possible differences in the variables affecting the school choice. We find that, although immigrants and chileans are similar, and their decisions are similar, they value certain characteristics differently, and the main reason behind the differences in school choice is related to the sociodemographic composition.
\end{abstract}

Key Words: Voucher system, immigrants, education. 


\section{INTRODUCCIÓN}

Durante el último tiempo la inmigración y su aumento han estado en el centro del debate público en Chile. En el año 2015 en nuestro país la cantidad de inmigrantes aumentó en un $80 \%$ con respecto al año 2005, llegando a un aproximado de 500.000. A pesar de que no es el país con la mayor tasa de inmigración en Latinoamérica, esta ola de inmigración es la mayor en su historia (Lupica, 2016). El tipo de inmigración en Chile es principalmente permanente. Alrededor de la mitad de los inmigrantes que ha llegado a Chile lo hace junto a sus hijos (Lafortune y Tessada, 2016). El enorme aumento de la inmigración permanente está ilustrado en el Gráfico 1, donde se presenta el número de visas de tipo permanente ${ }^{1}$ entregadas desde el año 2005 al año 2016.

El Gráfico 1 muestra que la cantidad de inmigrantes adultos se ha casi cuadruplicado, mientras que la cantidad de inmigrantes menores de edad, infantes, niños y adolescentes ha aumentado en casi un $260 \%$, siguiendo la misma tendencia.

Figura 1. Evolución de visas entregadas por año

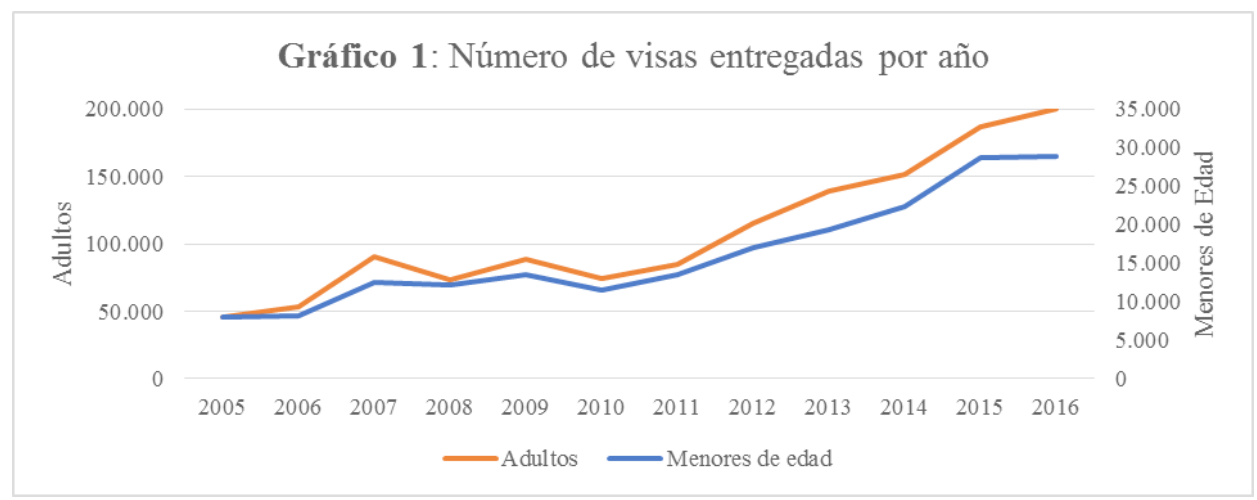

Fuente. Elaboración propia a partir de estadísticas migratorias, Extranjería, Gobierno de Chile.

Sin embargo, poco se sabe respecto a cómo los padres inmigrantes deciden el colegio en que matriculan a sus hijos, qué características de los colegios son más importantes para ellos y cómo se adecúan al sistema de elección de colegios en Chile.

En la actualidad hay numerosos estudios en Estados Unidos y Europa que abordan diversos aspectos de la relación entre inmigración y educación. Estos trabajos han desarrollado temas que van desde la adaptación del estudiante inmigrante (Cahan, Davis y Staub, 2001; Schwarz y Stiefel, 2006) hasta su desempeño académico en el país al que arriban (Glick y Hohmann-Marriot, 2007).

Sin embargo, el sistema voucher chileno hace especialmente interesante estudiar la decisión de los padres al momento de elegir un establecimiento educacional. Este sistema

\footnotetext{
Las visas de tipo permanente en Chile corresponden a las visas de permanencia definitiva, sujeta a contrato de trabajo, estudiante y "temporaria", todas asociadas a estudios o razones laborales y se otorgan por un mínimo de un año.
} 
se basa en la idea de que cada niño puede matricularse en el colegio que desee (Arverseth, 2014). En muchos otros países, la decisión de escolaridad está ligada a la ubicación geográfica. Chile, al separar la decisión educativa de la ubicación geográfica, permite enfocar el análisis en la decisión familiar de qué establecimiento educacional escoger. Para otros países esta decisión puede verse afectada por los valores inmobiliarios o el mercado laboral, lo que dificulta enormemente los análisis y estudios de la decisión escolar. Por lo tanto, el caso chileno nos permite enfocarnos en qué características del establecimiento son importantes para la elección de los padres. Adicionalmente, podemos estudiar esta elección de forma separada tanto para chilenos como para inmigrantes y comparar cómo las distintas variables afectan la decisión de cada tipo de familia.

Es importante entender las posibles fuentes de diferencia en la decisión escolar entre familias de chilenos e inmigrantes. Las familias pueden tener formaciones, características culturales o valoraciones por características escolares distintas. Por ejemplo, es posible que inmigrantes, al llegar al nuevo país, quieran estar cerca de compatriotas y formar redes con ellos, por lo tanto, matriculen a sus hijos en colegios con más inmigrantes (Pamuk, 2004) o bien; es posible que sientan que puede haber una estigmatización hacia los inmigrantes y prefieran no formar redes con ellos y matriculen a sus hijos en colegios con menos inmigrantes (Byrne, McGinnity, Smyth y Darmody, 2010; Söderström y Uusitalo, 2010). Por otro lado, es posible que los inmigrantes no estén familiarizados con las características que son importantes para los padres chilenos, como la prueba SIMCE (Chumacero, Gómez y Paredes, 2011; Gallego y Hernando, 2009) por lo que sus decisiones de establecimiento no consideren variables importantes para las familias chilenas o, dado el sistema voucher, sí intenten usar el máximo de la información disponible y, por lo tanto, elegir de forma similar a las familias chilenas.

El objetivo de este trabajo es responder a la pregunta: ¿Es la elección del colegio distinta para chilenos e inmigrantes? Y si es así, ¿en qué radican esas diferencias? En el caso de encontrar diferencias, buscamos entender qué factores sociodemográficos y del establecimiento son los considerados en forma distinta para chilenos y extranjeros.

Para lograr este objetivo se estimará un modelo de probabilidad de elección de establecimiento educacional. Esto es posible al utilizar datos administrativos del Ministerio de Educación de Chile y datos de la nacionalidad de todos los estudiantes matriculados en Chile. Lo anterior se complementa con información proveniente de las encuestas SIMCE para crear una base de datos rica en información del alumno, su familia, su establecimiento educacional y su entorno.

\section{REVISIÓN DE LITERATURA}

\subsection{EL SISTEMA VOUCHER EN CHILE}

El sistema de elección escolar aplicada en Chile en el año 1981, que fue inspirado por el trabajo de Milton Friedman (Friedman, 1955), consiste en la entrega a padres de un voucher por estudiante, que les otorga el derecho a matricular a su hijo en el establecimiento de su preferencia -público o privado- (Arverseth, 2014). Este programa permite la entrada de nuevos establecimientos al sector, busca que compitan y genera movimientos de estudiantes desde establecimientos de menor calidad a otros de mayor calidad (Epple y Romano, 1998). 
La teoría indica que los establecimientos, al competir, deberían aumentar su calidad, ya que los padres eligen los colegios de mejor calidad, pero también que se ajuste mejor a sus valores. De esta forma, los colegios intentarán satisfacer las demandas específicas de proyectos educativos de los padres más allá de una sola dimensión como calidad, introduciendo otras dimensiones en su oferta, de manera de atraer a padres que prioricen cierto conjunto de características (Arverseth, 2014; Hsieh y Urquiola, 2006). Los establecimientos de baja calidad y que no se ajusten a las demandas de los padres recibirán menos estudiantes (vouchers) y serán forzados a salir del mercado.

En Chile coexisten tres tipos de establecimientos: (1) los públicos (municipales), de financiamiento y administración pública (solo vouchers); (2) privados, de administración y financiamiento privado y (3) particulares subvencionados, de financiamiento compartido (vouchers y aportes de los padres) y administración privada.

Los establecimientos particulares subvencionados en Chile deben cumplir los mismos estándares impuestos por el Ministerio de Educación en términos de malla curricular y presupuesto que los establecimientos municipales. Sin embargo, los establecimientos con copago cuentan con libre entrada al mercado y libertad de utilizar requisitos de matrícula y sistemas de admisión para sus potenciales estudiantes. Estos establecimientos están significativamente menos regulados que los municipales (Arverseth, 2014). En particular, compiten por tipo de alumno, de manera de intentar atraer a los mejores alumnos y obtener mejores resultados en pruebas estandarizadas. En casos como este, la selección por parte de los establecimientos estaría conduciendo a la segregación (Elacqua, 2012).

Una posible consecuencia del sistema voucher, que no fue vista por la teoría, se refiere a la posible segregación del sistema. Para poder mantenerse competitivos, reducir costos y mejorar utilidades, los establecimientos tienden a atraer a los mejores alumnos ya que estos demandan menores recursos (Epple y Romano, 1998). Estudios han encontrado que la diversidad de matrícula cambia con el tipo de financiamiento del establecimiento. Los padres priorizan proyectos educativos con estudiantes de características similares a las propias lo que genera establecimientos segregados. Esto conduce a que padres con hijos de menor habilidad terminan matriculando a sus hijos en establecimientos públicos una vez que los padres de hijos con mayor habilidad y/o mayor ingreso hayan matriculado a sus hijos en establecimientos particulares subvencionados (Elacqua, 2012; Epple y Romano, 1998; Hsieh y Urquiola, 2006).

\subsubsection{Determinantes de la elección escolar}

La literatura de la elección escolar ha estudiado esta decisión desde muchos paradigmas distintos, lo que ha llevado a múltiples conjuntos de variables que pueden afectar la decisión de qué establecimiento escoger. Por ejemplo, se ha estudiado los efectos de la productividad, segregación, cultura y otros aspectos determinantes. Nosotros nos enfocaremos en las determinantes de la decisión de los padres dado el sistema voucher existente en Chile. La literatura ha identificado varias características que los padres observan para tomar su decisión.

La calidad del establecimiento es una determinante importante. A pesar de que no es posible asegurar que padres, educadores y establecimientos estén de acuerdo en qué se define como calidad (Bosetti, 2004), todos, independiente del estrato socioeconómico o etnia de la familia, declaran que lo más importante para ellos es 
la calidad del establecimiento educacional que eligen para sus hijos (Kleitz, Wehier, Tedin y Matland, 2000). Sin embargo, debido a la multidimensionalidad del problema se encuentran diferencias entre lo que los padres declaran y los establecimientos que efectivamente eligen.

La multidimensionalidad de la decisión ha sido documentada por varios autores. Por ejemplo, Weiher y Tedin (2002) encuentran en Texas que más de la mitad de los padres declaran que el aspecto más importante para ellos es la calidad, medida en el resultado del establecimiento y el puntaje del colegio en pruebas estandarizadas, pero al tener acceso a un sistema de voucher cambian a sus hijos a establecimientos privados con puntajes promedio en pruebas estandarizadas considerablemente menores a los que asistían previamente. En Chile se encuentran resultados similares respecto a la prueba SIMCE (Gallego y Hernando, 2009).

Lo anterior puede estar asociado a las características de las familias. Así, padres de mayor estrato socioeconómico, con años de educación y mayores expectativas de sus hijos, suelen matricularlos en establecimientos superiores en términos de puntajes en pruebas estandarizadas; mientras que padres de menor estrato socioeconómico y menos años de educación suelen matricular a sus hijos en el establecimiento más cercano al hogar (Gallego y Hernando, 2009; Hastings, Kane y Staiger, 2005). Tomando esto en cuenta, ciertos estudios proponen que la segregación entre establecimientos públicos y particulares subvencionados tiene parte de su origen en la elección de los mismos padres, los que se "auto-segregarían". En la práctica, se puede ver que los padres matriculan a sus hijos en establecimientos con alumnos de características demográficas similares a ellos y no necesariamente en establecimientos con los mejores puntajes en pruebas estandarizadas (Elacqua, Schneider y Buckley, 2006; Chumacero et al., 2011; Hastings y Weinstein, 2007; Nishimura y Yamano, 2013).

\subsubsection{La elección de inmigrantes}

A pesar de que no existen estudios relacionados con la elección escolar de estudiantes inmigrantes en Chile, es posible encontrar tales estudios en el mundo. Estos se enfocan en estudiar los efectos de la inmigración en la educación. En particular, existen dos estudios que abordan la elección escolar de padres para sus hijos tanto para inmigrantes como para nativos: Mavisakalyan (2012) y Murray (2016) estudian la fuga de estudiantes nativos (Australia y Estados Unidos, respectivamente) desde colegios públicos a privados frente a la llegada de inmigrantes. En ambos trabajos se analizan tanto los determinantes y variables que afectan la elección de inmigrantes como de nativos. Ambos estudios encuentran que los nativos responden a un flujo de inmigrantes "lejanos" y cambian a sus hijos de colegios públicos a colegios privados, entendiéndose inmigrantes lejanos como aquellos culturalmente distintos y/o que hablan otro idioma. Al encontrarse con inmigrantes "cercanos" (culturalmente similares) o de mayor nivel socioeconómico la fuga es menor. Además, se encuentra que el efecto es más pronunciado en zonas con pocos habitantes que no están acostumbrados a recibir inmigrantes.

Nuestro trabajo utiliza la misma estrategia empírica para analizar la decisión, pero se enfoca en las potenciales diferencias en la decisión de a qué establecimiento matricular, en lugar del impacto que esta decisión tiene sobre los nativos. 


\section{METODOLOGÍA EMPÍRICA, DATOS Y VARIABLES}

\subsection{METODOLOGÍA EMPÍRICA}

De acuerdo con lo discutido en la revisión de literatura, debemos estimar un modelo de elección escolar que incluya características del establecimiento, características sociodemográficas del estudiante y su familia. Se estimarán modelos separados para estudiantes chilenos e inmigrantes. Al realizar las estimaciones por separado podremos observar y comparar las diferencias en la importancia relativa de las variables antes mencionadas en las decisiones de ambos grupos. De esta forma, el modelo permite que la importancia de los determinantes varíe de acuerdo con las características particulares de cada grupo.

El enfoque empírico se sustenta en un modelo teórico que asume que los padres maximizan su bienestar (utilidad) al elegir un establecimiento para sus hijos. Esta utilidad depende de sus características sociodemográficas y las características del establecimiento educacional. En resumen:

$$
u_{i j}^{*}=x_{i j} \beta+w_{i j} \gamma+\varepsilon_{i j}
$$

El subíndice $i$ representa a la familia ${ }^{2}$ y el subíndice $j$ el establecimiento educacional. Donde $x_{i j}$ representa características familiares y del alumno como edad, género, educación de la madre e ingreso per cápita. $w_{i j}$ representa características del establecimiento como calidad, medida como puntaje SIMCE, promedio de educación de los padres en el establecimiento y cantidad de requisitos solicitados al momento de postular.

Cabe destacar que el objetivo de este modelo no es analizar la decisión de matrícula en un establecimiento en particular, sino cómo las distintas características familiares y del establecimiento afectan la decisión a nivel poblacional. En la actualidad, en Chile, la característica más relevante es el aporte financiero de los padres (copago) al establecimiento. Por lo tanto, nos enfocaremos en la decisión entre establecimientos municipales y particulares subvencionados.

Para analizar esta decisión, definimos la variable $u_{i j}$ de la siguiente manera: Si los padres eligen un establecimiento municipal, la variable $u_{i j}$ tomará valor 1 y cero si el niño está matriculado en un establecimiento particular subvencionado.

El uso de un modelo de probabilidad no lineal con distribución normal del error ha sido frecuentemente usado en la literatura, existen variados trabajos que estiman modelos de elección escolar tradicionales y para inmigrantes y nativos, tal como Mavisakalyan (2012), Chumacero et al. (2011), García y Paredes (2010), Gallego (2006), Betts y Fairlie (2003), Angrist et al. (2002), Cox y Jiménez (1990) y Long y Toma (1988).

Es importante notar que algunas de las variables utilizadas para estimar el modelo fueron rezagadas. Esto se debe a que la decisión de qué colegio elegir, tanto para inmigrantes como para chilenos, no se tomó el año en que obtuvimos los datos del estudiante, que en este trabajo corresponde al año 2012 al 2016, sino antes de matricularse en el colegio. Los padres eligen colegios para sus hijos al menos un año antes de su ingreso. Por lo tanto, los valores que observaron ellos al decidir no fueron los mismos que los valores del año en que

Para simplificar el modelo supondremos solo un hijo por familia. 
el estudiante rinde el SIMCE, sino de un año anterior. La información rezagada ayuda a disminuir este potencial problema estadístico ${ }^{3}$.

\subsection{DATOS}

Las bases de datos utilizadas en este trabajo corresponden a la Matrícula única Oficial 20122016 del Ministerio de Educación, las encuestas de padres Simce 2012-2016 para obtener características personales y familiares del estudiante y el Directorio de Establecimientos educacionales 2012-2016 del Ministerio de Educación. Para las variables de SIMCE rezagadas se utilizaron las bases de puntaje SIMCE desde el año 2004-2015.

Consideramos en nuestro análisis características individuales, familiares y del establecimiento. La literatura ha encontrado que estas variables son importantes para la decisión de estudiantes nativos e inmigrantes en el caso de estudios chilenos e internacionales.

Dentro de las variables utilizadas se incluyen características individuales del estudiante, utilizamos género y una variable binaria que indica si el estudiante es inmigrante o no. Utilizamos características familiares tales como ingreso del hogar, años de educación de la madre y del padre, número de requisitos solicitados al momento de la postulación al establecimiento y una variable binaria que indica si el hogar se encuentra en la misma comuna que el establecimiento educacional ${ }^{4}$. Las características a nivel de establecimiento educacional incluyen proporción de estudiantes inmigrantes en el establecimiento (por cuartiles $^{5}$ ), grupo socioeconómico del establecimiento e indicador del promedio SIMCE rezagado ${ }^{6}$. Entre estos últimos se incluyen requisitos tales como prueba de ingreso, certificado de remuneraciones, entrevista con los padres o certificados de notas de establecimiento anterior. Además de esto, se incluyen controles de efectos fijos por región para poder capturar las diferencias regionales de atender a un colegio que no puedan ser explicadas por los controles.

\subsection{ESTADÍSTICOS DESCRIPTIVOS}

Una posible explicación de las diferencias en la elección entre inmigrantes y chilenos se puede deber a que la composición sociodemográfica sea distinta entre ambos grupos, por lo que es importante observar qué tan distintos son los inmigrantes de los chilenos. Sería de esperar que, de tener composiciones diversas, la decisión de establecimiento también difiera. Por lo tanto, presentamos estadísticas descriptivas para analizar qué tan distintos son los chilenos y los inmigrantes y qué tan variadas son sus decisiones.

Se estimaron regresiones con variables rezagadas y sin rezagar y estas no mostraron diferencias significativas. Finalmente se decidió trabajar con las variables rezagadas debido a que estas son una mejor aproximación al modelo ideal.

4 Esta variable puede ser considerada como una variable de proximidad y relacionada con la distancia al establecimiento.

5 En particular, la proporción de alumnos inmigrantes se calcula con cuatro variables correspondientes al cuartil de proporción de inmigrantes en el establecimiento. Cada una toma valor 1 si el establecimiento se encuentra dentro de un cuartil determinado.

6 Corresponde al SIMCE del establecimiento dividido en el promedio SIMCE todos los establecimientos de su misma dependencia en el país, sin contar el propio. 
Cabe destacar que estudios han encontrado que la decisión de los padres por un establecimiento particular subvencionado está positivamente correlacionada con el ingreso del hogar, indicando que padres de mayor ingreso tienen más probabilidades de matricular a sus hijos en establecimientos particulares subvencionados (Contreras, Sepúlveda y Bustos, 2010; Elacqua y Fabrega, 2004). El Gráfico 2 muestra la distribución de estudiantes inmigrantes y chilenos por dependencia de establecimiento. Es interesante observar que más de la mitad de los estudiantes chilenos se encuentran concentrados en los establecimientos particulares subvencionados, mientras que cerca de la mitad de los estudiantes inmigrantes se encuentran matriculados en establecimientos públicos. Dado que la literatura indica que estudiantes de mayor ingreso tienden a matricularse en establecimientos particulares subvencionados (Elacqua et al. 2006; Gallego y Hernando, 2009), una posible explicación de lo observado es que los estudiantes inmigrantes provengan de familias de menor ingreso.

Figura 2. Distribución proporcional

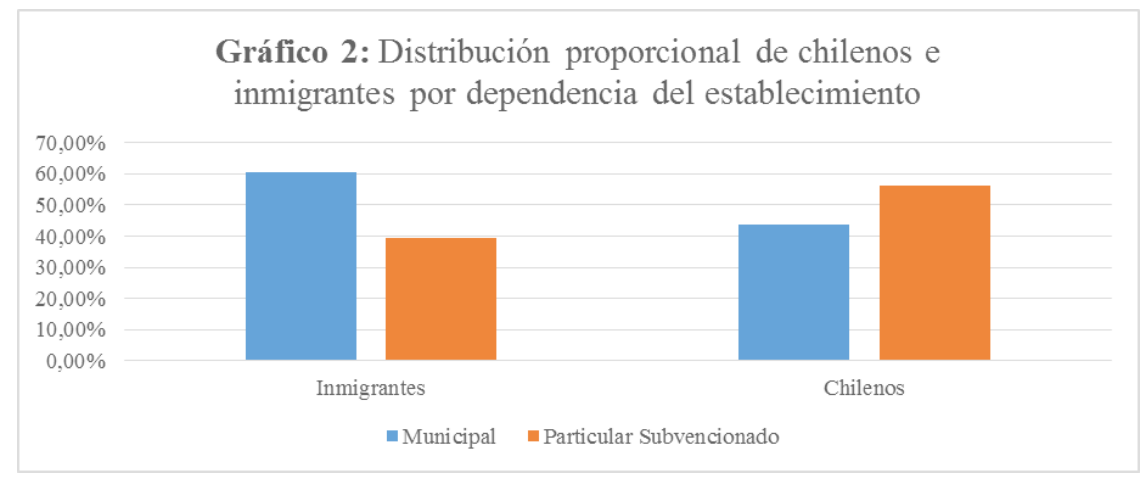

Fuente. Elaboración propia a partir de datos administrativos Mineduc, Gobierno de Chile.

El Gráfico 3 presenta la distribución del ingreso para inmigrantes y chilenos por percentiles de ingreso, para analizar si efectivamente el ingreso puede explicar las diferencias observadas en el Gráfico 2. El Gráfico 3 muestra que las proporciones de inmigrantes y chilenos en general no difieren a lo largo de la distribución del ingreso con excepción del último cuartil. Por lo que no hay evidencia para concluir que la distribución del ingreso es capaz de explicar la diferencia de decisión de establecimiento presentada en el Gráfico 2.

El Gráfico 4 muestra la distribución de los percentiles de años de educación de la madre. Esto se debe a que diferencias en la educación de los padres podría ser otra posible explicación para lo observado en el Gráfico 2. Al igual que en el caso de la distribución del ingreso no hay diferencias significativas entre inmigrantes y chilenos. Luego, la evidencia indica que las diferencias educacionales entre familias inmigrantes y chilenas no puede explicar las diferencias observadas en el Gráfico 2. Por lo tanto, es necesario estudiar con más detalle la forma en que las familias están tomando la decisión de establecimiento educacional. 
Figura 3. Gráficos de percentil ingresos y educación de la madre
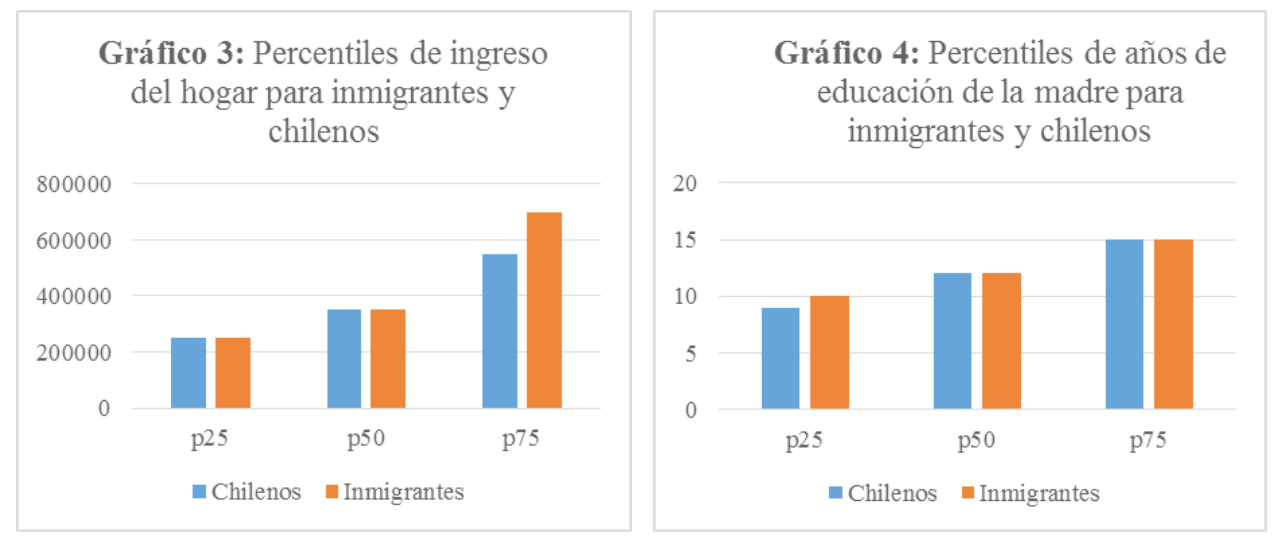

Fuente. Elaboración propia a partir de encuesta de padres SIMCE 2012-2016.

Agencia de calidad de la Educación.

\section{RESULTADOS}

De acuerdo con lo encontrado en la literatura, variables como calidad, tamaño de clase, composición racial y socioeconómica, así como cercanía al hogar; son relevantes para la decisión de los padres. Por lo mismo, se espera que la calidad del establecimiento aumente la probabilidad de que el estudiante se matricule en el colegio, y que a medida que se incremente la educación de la madre, decrezca la probabilidad de que el estudiante se matricule en un establecimiento municipal y que los requisitos solicitados al postular al colegio mermen la probabilidad de que el estudiante se matricule en dicho establecimiento (Chumacero et al., 2011; Elacqua et al., 2006; Gallego y Hernando, 2009; Kleitz et al., 2000; Nishimura y Yamano, 2013).

La Tabla 1 muestra las estimaciones de nuestro modelo estadístico descrito anteriormente. Es decir, se calcula la probabilidad de la decisión de elegir un establecimiento municipal de forma separada para estudiantes chilenos e inmigrantes. La primera columna de la tabla presenta los efectos marginales del modelo para extranjeros, mientras la segunda muestra los efectos marginales del modelo para chilenos ${ }^{7}$. Además, aquellas variables presentadas en negrita son estadísticamente diferentes entre modelos ${ }^{8}$.

Dado que los establecimientos particulares subvencionados tienen en promedio mejores resultados en pruebas estandarizadas (Tokman, 2005), es esperable que padres con más años de educación y/o que tengan acceso a mejor información, prefieran matricular a sus hijos en establecimientos particulares subvencionados. La Tabla 1 muestra un signo negativo en la probabilidad de que los padres con mayor educación matriculen a sus hijos en establecimientos municipales. Lo anterior es consistente con la literatura

En el caso de los modelos tipo probit el coeficiente de interés en el análisis es el coeficiente marginal. Los coeficientes directamente de la estimación del modelo están disponibles para el lector interesado.

8 Se utiliza el test de Wald para estudiar la diferencia estadística entre los coeficientes. 
chilena que encuentra que la educación de la madre y la matrícula en colegios públicos tiene una correlación negativa (Contreras et al., 2010; Elacqua y Fabrega, 2004). Los años de educación y capital humano están positivamente correlacionados con el salario (Ashenfelter y Rouse, 1999) y, en consecuencia, es más probable que padres de mayor ingreso matriculen a sus hijos en establecimientos particulares subvencionados. Además, la literatura dice que padres con mayor educación tienen más expectativas de sus hijos y a su vez, estos matriculan con mayor probabilidad a sus hijos en colegios privados (Galab, Vennam, Komanduri, Benny y Georgiadis, 2013; Gallego y Hernando, 2009). Lo interesante es que este resultado es más marcado para estudiantes chilenos que inmigrantes cuando nos enfocamos en educación de la madre.

Tabla 1. Resultados del probit estimando las variables que afectan la elección de un establecimiento municipal para chilenos e inmigrantes

\begin{tabular}{|c|c|c|}
\hline & (1) & (2) \\
\hline VARIABLES & $\begin{array}{l}\text { Ef. Marginal } \\
\text { Extranjeros }\end{array}$ & $\begin{array}{l}\text { Ef. Marginal } \\
\text { Chilenos }\end{array}$ \\
\hline \multirow[t]{2}{*}{ Educación de la madre (Años) } & $-0.002 * *$ & $-0.006 * * *$ \\
\hline & $(0.001)$ & $(0.0001)$ \\
\hline \multirow[t]{2}{*}{ Educación del Padre (Años) } & $-0.002 * *$ & $-0.003 * * *$ \\
\hline & $(0.001)$ & $(0.0001)$ \\
\hline \multirow[t]{2}{*}{ Mujer } & $-0.011 *$ & $-0.010 * * *$ \\
\hline & $(0.007)$ & $(0.001)$ \\
\hline \multirow[t]{2}{*}{ Alumno estudia en la misma comuna en que vive } & $0.111 * * *$ & $0.054 * * *$ \\
\hline & $(0.009)$ & $(0.001)$ \\
\hline \multirow[t]{2}{*}{ Requisitos de postulación } & $-0.040 * * *$ & $-0.043 * * *$ \\
\hline & $(0.002)$ & $(0.0003)$ \\
\hline \multirow[t]{2}{*}{ Inmigrantes por colegio 2} & -0.031 & $-0.032 * * *$ \\
\hline & $(0.030)$ & $(0.001)$ \\
\hline \multirow[t]{2}{*}{ Inmigrantes por colegio 3} & 0.018 & $0.050 * * *$ \\
\hline & $(0.028)$ & $(0.001)$ \\
\hline \multirow[t]{2}{*}{ Inmigrantes por colegio 4} & $0.336 * * *$ & $0.267 * * *$ \\
\hline & $(\mathbf{0 . 0 2 7})$ & $(\mathbf{0 . 0 0 1})$ \\
\hline \multirow[t]{2}{*}{ Indicador Simce } & $1.283 * * *$ & $1.042 * * *$ \\
\hline & $(0.046)$ & $(\mathbf{0 . 0 0 5})$ \\
\hline \multirow{2}{*}{ Grupo Socioeconómico establecimiento $=$ Medio bajo } & $-0.110 * * *$ & $-0.056 * * *$ \\
\hline & $(\mathbf{0 . 0 1 1})$ & $(\mathbf{0 . 0 0 1 )}$ \\
\hline
\end{tabular}




\begin{tabular}{|l|c|c|}
\hline \multirow{2}{*}{ Grupo Socioeconómico establecimiento = Medio } & $-0.341 * * *$ & $-0.405 * * *$ \\
\cline { 2 - 3 } & $(0.011)$ & $(0.001)$ \\
\hline \multirow{2}{*}{ Grupo Socioeconómico establecimiento = Medio alto } & $\mathbf{- 0 . 6 8 7 * * *}$ & $\mathbf{- 0 . 6 2 3} * * *$ \\
\cline { 2 - 3 } & $\mathbf{( 0 . 0 1 3 )}$ & $\mathbf{( 0 . 0 0 1 )}$ \\
\hline Grupo Socioeconómico establecimiento = Alto & $\mathbf{- 0 . 8 1 9 * * *}$ & $\mathbf{- 0 . 6 7 5} * * *$ \\
\hline Constante & $\mathbf{( 0 . 0 2 0 )}$ & $\mathbf{( 0 . 0 0 2 )}$ \\
\hline & & \\
\hline & & \\
\hline Observaciones & & $2,451,759$ \\
\hline
\end{tabular}

Errores estándar en paréntesis. $* * * \mathrm{p}<0.01, * * \mathrm{p}<0.05, * \mathrm{p}<0.1$.

Fuente. Construcción propia a partir de los datos de matrícula oficial del Ministerio de Educación de Chile y Encuestas Simce nivel alumno de la Agencia de calidad de la Educación.

La variable dependiente es "municipal", binaria con valor 1 si el alumno está matriculado en un colegio municipal y valor 0 si está matriculado en un colegio particular subvencionado. "Educación de la Madre" = Años de educación de la madre, toma valores entre 0 y 23. "Educación del Padre" = Años de educación del padre, toma valores entre 0 y 23. "Mujer" = variable binaria con valor 1 si el individuo es mujer y 0 si es hombre. "Alumno estudia en la misma comuna en que vive" = variable binaria con valor 1 si el alumno vive en la misma comuna en que se ubica su establecimiento educacional y 0 en caso contrario. "Requisitos de postulación" = Indica el número de requisitos de postulación que se solicitaron a la familia al momento de la matrícula y toma valores de 1 al 11 . "Inmigrantes por colegio 2" = variable binaria con valor 1 si la proporción de alumnos inmigrantes en el establecimiento corresponde al segundo cuartil de inmigrantes por establecimiento, esto es entre $0.054 \%$ y $0.27 \%$ de inmigrantes en la matrícula. "Inmigrantes por colegio 3" = variable binaria con valor 1 si la proporción de alumnos inmigrantes en el establecimiento corresponde al tercer cuartil de inmigrantes por el establecimiento, entre $0.27 \%$ y $0.8 \%$ de inmigrantes en la matrícula. "inmigrantes por colegio 4 " = variable binaria con valor $1 \mathrm{si}$ la proporción de alumnos inmigrantes en el establecimiento corresponde al último cuartil de inmigrantes por establecimiento, esto es más de $0.8 \%$ de inmigrantes en la matrícula. "indicador SIMCE" = un indicador de SIMCE que corresponde al SIMCE del establecimiento dividido en el promedio SIMCE todos los establecimientos de su misma dependencia en el país, sin contar el propio. "Grupo socioeconómico del establecimiento= Medio Bajo" = variable binaria con valor 1 si el grupo socioeconómico del establecimiento es medio bajo. "Grupo socioeconómico del establecimiento= Medio" = variable binaria con valor 1 si el grupo socioeconómico del establecimiento es medio. "Grupo socioeconómico del establecimiento= Medio alto" = variable binaria con valor 1 si el grupo socioeconómico del establecimiento es medio alto. "Grupo socioeconómico del establecimiento= alto" = variable binaria con valor 1 si el grupo socioeconómico del establecimiento es alto. También se controla por región e ingreso del hogar.

Otra potencial fuente de diferencia entre inmigrantes y chilenos son los requisitos de postulación. En nuestros datos podemos medir el número de requisitos solicitados al momento de postular. Estas variables pueden ser importantes porque inmigrantes y chilenos pueden diferir en la facilidad que tienen las familias para reunir estos requisitos. Cabe hacer notar que algunos de estos pueden ser obtenidos en línea por un chileno, pero no necesariamente por inmigrantes ${ }^{9}$. Es por lo mismo que esta variable en particular es muy interesante para estudiar las potenciales diferencias entre chilenos en inmigrantes. La Tabla 1 muestra que el número de requisitos de postulación que el colegio solicita al momento

9 Como, por ejemplo, certificado de nacimiento, certificado de notas del establecimiento anterior o certificado de remuneraciones. 
de matricular al estudiante en el colegio es estadísticamente significativo y negativo tanto para chilenos como para inmigrantes. Es decir, tanto los primeros como los inmigrantes no prefieren establecimientos que piden un alto número de requisitos. Particularmente, la diferencia no es estadísticamente significativa entre ambos grupos. Esto tal vez se debe a que los padres eligen el establecimiento en el que saben que su hijo(a) va a ser aceptado. Por lo tanto, ya sea los primeros como los segundos realizan una elección similar.

Para saber si los coeficientes entre modelos son estadísticamente diferentes realizamos un test de Wald y así comparar coeficientes entre regresiones. Marcamos con letra negrita las variables cuando los coeficientes son significativamente distintos entre ambos modelos con una significancia del 10\%. De las variables de interés, las que presentan una diferencia estadísticamente significativa son las variables de educación de la madre que es alumno y estudia en la misma comuna que vive, proporción de alumnos inmigrantes, puntaje SIMCE y grupo socioeconómico del establecimiento. Esto significa un coeficiente mayor para chilenos en el caso de la educación de la madre y coeficientes mayores para extranjeros de la comuna en que viven, último cuartil de inmigrantes por colegio, SIMCE y grupo socioeconómico.

Respecto a la proporción de alumnos extranjeros en el establecimiento, existen variadas explicaciones para un coeficiente tanto positivo como negativo. Por un lado, es probable que inmigrantes prefieran ir a colegios con otros inmigrantes y formar clusters entre ellos. Otra posibilidad es que exista segregación en el sistema escolar chileno, por lo que los inmigrantes se encuentren en el mismo colegio al no ser aceptados en ningún otro establecimiento. En ambos casos el coeficiente de la variable proporción de alumnos inmigrantes será positivo. Por otro lado, es posible que, debido a la discriminación y estigmatización de los inmigrantes estos prefieran no encontrarse con otros extranjeros y no formar clusters entre ellos, caso en el que el coeficiente de proporción de alumnos inmigrantes sería negativo. Si los chilenos prefieren matricular a sus hijos en colegios con una menor cantidad de inmigrantes, se espera que el coeficiente de proporción de alumnos inmigrantes sea negativo. Sin embargo, también es posible que inmigrantes y chilenos elijan de la misma forma en caso de que no exista ni información incompleta, ni segregación ni discriminación, por lo que el coeficiente simplemente estaría captando la correlación de padres que eligen el mejor colegio para sus hijos en cuyo caso sería positivo. La literatura en general encuentra coeficientes negativos que indican que a medida que aumenta la proporción de alumnos inmigrantes en un establecimiento la probabilidad de matrícula de un estudiante nativo disminuye. Sobre todo, en el caso de inmigrantes "lejanos", en otras palabras, de culturas diferentes, estatus socioeconómico diferente y que hablan diferentes idiomas (Mavisakalyan, 2012; Murray, 2016).

Para entender mejor qué tan importante es el número de inmigrantes en la decisión de los padres, creamos cuatro variables dummies para describir la proporción de inmigrantes en el colegio. En particular, se dividió la proporción en sus cuatro cuartiles, siendo el primer cuartil (pocos inmigrantes) la variable omitida en la regresión. En todos los casos los inmigrantes prefieren establecimientos con más inmigrantes que el primer cuartil de inmigrante o son indiferentes ${ }^{10}$. Además, a medida que aumentan los inmigrantes por establecimiento, aumenta el efecto marginal. Esto significa que los inmigrantes prefieren consistentemente establecimientos con un alto número de inmigrantes, lo que está en

10 Como lo es el caso del segundo cuartil, cuyo coeficiente no es estadísticamente significativo y por lo tanto puede ser entendido como indiferencia entre establecimientos del primer o segundo cuartil. 
línea con la literatura de clusters. Respecto a los coeficientes para chilenos encontramos evidencia mixta. Los chilenos tienden a no preferir establecimientos con un bajo número de inmigrantes, pero muestran una preferencia por establecimientos con un alto número de inmigrantes. Sin embargo, esta probabilidad es menor que la de inmigrantes, por lo que puede estar relacionado a que inmigrantes y chilenos eligen de forma similar los establecimientos buenos y ese coeficiente estaría captando una correlación.

Los padres de estudiantes nativos podrían estar reaccionando frente a la llegada de inmigrantes "lejanos". Luego, analizamos la composición y procedencia de las familias extranjeras que llegan a Chile. Entendemos inmigrantes lejanos, en este caso, a inmigrantes que no hablan el idioma español y provienen de culturas fundamentalmente distintas (tal como lo sería la cultura asiática, por ejemplo). Esta distinción es importante, ya que la literatura ha encontrado que familias nativas prefieren evitar establecimientos con una proporción alta de inmigrantes lejanos. Este resultado no se mantiene al analizar inmigrantes cercanos.

Tomando esto en cuenta, analizamos la composición y procedencia de las familias extranjeras que llegan a Chile. Los estadísticos descriptivos ya analizados anteriormente nos muestran que, en promedio, los inmigrantes en Chile no difieren mucho de los chilenos en términos de ingreso y educación de la madre. Usando los datos de las visas de permanencia definitiva otorgadas, mostramos en la Tabla 2 la procedencia de los inmigrantes en los últimos 10 años. Se observa que los inmigrantes provienen principalmente de Perú, Bolivia, España y Venezuela, todos ellos de habla hispana. De hecho, de los 10 principales países de procedencia, solo 2 hablan otra lengua. Luego, no es aplicable en este caso el efecto de los inmigrantes lejanos en la decisión de los padres y podemos interpretar la preferencia de chilenos por establecimientos con una alta proporción de inmigrantes como una correlación. Es decir, inmigrantes y chilenos coinciden en cierta medida en sus preferencias por establecimientos.

Tabla 2. Número de visas de residencia definitiva otorgadas por nacionalidad entre 2005 y 2016

\begin{tabular}{|l|c|}
\hline \multicolumn{1}{|c|}{ País de procedencia } & Número de visas \\
\hline Perú & 123670 \\
\hline Bolivia & 43750 \\
\hline España & 9027 \\
\hline Venezuela & 8543 \\
\hline China República Popular & 7571 \\
\hline Estados Unidos & 4712 \\
\hline Cuba & 3865 \\
\hline Uruguay & 3523 \\
\hline México & 3114 \\
\hline Francia & 2830 \\
\hline
\end{tabular}

Fuente. Elaboración propia a partir de datos de visas de tipo permanente del departamento de Extranjería. 
Al comparar los efectos marginales de ambos modelos nos encontramos con que no es posible detectar diferencias significativas entre ellos. Es importante notar que las composiciones de las muestras son ligeramente distintas. Por lo tanto, los efectos marginales no son directamente comparables. Esto se debe a que los efectos marginales de cada modelo están evaluados en distintos puntos de la distribución, lo que nos lleva a mezclar el efecto composición con el efecto elección. Para poder realizar un análisis adecuado de las magnitudes de los efectos marginales se evalúan los efectos marginales de cada regresión en las medias de ambos grupos, tanto de chilenos como inmigrantes. Luego, calculamos la probabilidad de que un padre inmigrante o chileno matricule a su hijo en un establecimiento municipal, usando los efectos marginales evaluados en los mismos puntos de la distribución. Esto se puede entender al observar la elección de chilenos y la elección de inmigrantes imponiendo que tienen las mismas características que los chilenos o al revés, determinar la elección de inmigrantes y de chilenos imponiendo que tienen las mismas características. La Tabla 3 muestra el cálculo de la probabilidad de matricular en un establecimiento municipal para los ejercicios descritos anteriormente.

Tabla 4. Probabilidad de matricularse en un colegio municipal

\begin{tabular}{|l|r|}
\hline Ef. Mg. chilenos en medias de chilenos & 0.3964727 \\
\hline Ef. Mg. chilenos en medias de extranjeros & 0.4261737 \\
\hline Ef. Mg. extranjeros en medias de extranjeros & .5651222 \\
\hline Ef. Mg. extranjeros en medias de chilenos & 0.36683 \\
\hline
\end{tabular}

Fuente. construcción propia a partir de los datos de matrícula oficial del Ministerio de Educación de Chile y Encuestas Simce nivel alumno de la Agencia de Calidad de la Educación. La probabilidad está construida a partir de las estimaciones en la Tabla

La Tabla 3 muestra que, al comparar la decisión de inmigrantes con chilenos, cuando usamos la composición de chilenos (filas 1 y 4), estos muestran una probabilidad más alta de matricular a sus hijos en un establecimiento municipal. Sin embargo, al evaluar la decisión de los padres usando la composición de los inmigrantes (filas 2 y 3), son aquellos los que muestran una preferencia por los establecimientos municipales.

Lo anterior nos indica que la composición sociodemográfica de las distintas poblaciones es el efecto más importante a la hora de evaluar las diferencias entre inmigrantes y chilenos. Es interesante destacar que cuando evaluamos la decisión de los extranjeros usando los atributos de la población chilena, estos muestran una menor tendencia a matricular a sus hijos en establecimientos municipales, lo que contrasta con lo presentado en el Gráfico 2, donde se observa que los inmigrantes parecieran preferir los establecimientos municipales. Esto es importante, porque muestra que, en términos de la decisión de establecimientos, los padres nativos y foráneos se comportan de manera similar, lo que habla de que el sistema voucher permite este accionar similar. Son las características de cada población las que generan las ligeras diferencias entre los grupos. 


\section{CONCLUSIONES Y RECOMENDACIONES}

En este trabajo estimamos un modelo de elección escolar para chilenos e inmigrantes con la intención de estudiar potenciales diferencias en su decisión al momento de elegir un establecimiento educacional. Evidencia preliminar indica que los segundos prefieren establecimientos municipales por sobre particulares subvencionados, a pesar de lo similares que son las poblaciones en ambos casos en términos de ingreso y educación ${ }^{11}$.

Usando un modelo de probabilidad no lineal, estudiamos cómo distintas características sociodemográficas de la familia y características del establecimiento pueden impactar la decisión. Realizamos estas estimaciones por separado para chilenos e inmigrantes. Encontramos que existen ligeras diferencias entre cómo las distintas variables afectan esta decisión. Por ejemplo, hallamos que una baja proporción de inmigrantes no desalienta la decisión por un establecimiento, pero sí lo hace para los nativos. Por otro lado, características como la calidad o la cercanía son valoradas de forma similar entre los dos grupos. La cantidad de requisitos, que es una potencial fuente de segregación, no afecta en forma diferente a ambos.

Finalmente, y para darle un sentido a las estimaciones del modelo de probabilidad, calculamos la probabilidad de elegir un establecimiento municipal para ambos grupos, pero imponiendo que tengan características similares. De esta manera, evitamos que las diferencias en la composición sociodemográfica afecten nuestro análisis. Estos cálculos nos indican que, al evaluar las decisiones en las mismas características poblacionales, los inmigrantes no muestran una clara preferencia por establecimientos municipales, y que el efecto de las diferencias en la composición sociodemográfica es posiblemente la explicación más importante al evaluar las diferencias observadas en la elección de establecimiento entre inmigrantes y chilenos.

Los hallazgos en este trabajo son significativos por varias razones. En primer lugar, se muestra que el sistema de voucher en Chile permite que extranjeros y chilenos escojan de manera similar, lo que indica que, hasta cierto punto, el sistema es ciego con respecto al origen del estudiante. Lo anterior evidencia que los padres tienden a informarse lo más rápido posible de las particularidades del sistema educativo, ya que no se ven diferencias sustantivas en cómo los inmigrantes escogen los establecimientos educacionales respecto a los chilenos.

Es muy importante seguir estudiando la calidad de la inserción de los extranjeros en Chile y generar políticas públicas en el caso de que sea necesario, debido a que la posibilidad de que exista segregación puede ser perjudicial para la integración social de estos estudiantes. Esto puede tener diversas implicancias en su rendimiento académico, experiencia educativa y resultados laborales posteriores.

\section{REFERENCIAS BIBLIOGRÁFICAS}

Angrist, J., Bettinger, E., Bloom, E., King, E. y Kremer, M. (2002). Vouchers for private schooling in Colombia: Evidence from a randomized natural experiment. American Economic Review, 92(5), 1535-1558.

11 Medida como educación de la madre. 
Estudios Pedagógicos XLV, Nº 2: 29-45, 2019

DIFERENCIAS EN LA ELECCIÓN DE ESTABLECIMIENTO EDUCACIONAL PARA LA POBLACIÓN LOCAL E

INMIGRANTES: CASO CHILENO

Arverseth, L. (2014). Friedman's school choice theory: The chilean education system. All graduate plan $B$ and other reports, (386).

Ashenfelter O. y Rouse C. (1999). Schooling, intelligence, and income in America: Cracks in the bell curve. National Bureau of Economic Research Working Paper Series, (6902).

Betts, J. y Fairlie, R. (2003). Does immigration induce "native flight" from public schools into private schools? Journal of Public Economics, 87(5-6), 987-1012.

Bosetti, L. (2004). Determinants of school choice: Understanding how parents choose elementary schools in Alberta. Journal of education policy, 19(4), 387-405.

Byrne D., McGinnity F., Smyth E. y Darmody, M. (2010). Immigration and school composition in Ireland, Irish Educational Studies, 29(3), 271-288.

Cahan, S., Davis, D. y Staub, R. (2001). Age at immigration and scholastic achievement in schoolage children: Is there a vulnerable age? International Migration Review, 35(134), 587-595.

Chumacero, R., Gómez, D. y Paredes, R. (2011). I would walk 500 miles (if it paid): Vouchers and school choice in Chile. Economics of Education Review, 30(5), 1103-1114.

Contreras, D., Sepúlveda, P., y Bustos, S. (2010). When schools are the ones that choose: The effects of screening in Chile. Social Science Quarterly, 91(5), 1349-1368.

Cox, D. y Jimenez, E. (1990). The relative effectiveness of private and public schools. Evidence from two developing countries. Journal of Development Economics, 34(1-2), 99-121.

Elacqua, G. (2012). The impact of school choice and public policy on segregation: Evidence from Chile. International Journal of Educational Development, 32(3), 444-453.

Elacqua, G. y Fabrega, R. (2004). El consumidor de la educación: El actor olvidado de la libre elección de escuelas en Chile. Santiago: Programa de Promoción de la Reforma Educativa en América Latina y el Caribe, PREAL.

Elacqua, G., Schneider, M. y Buckley, J. (2006). School choice in Chile: Is it class or the classroom? Journal of Policy Analysis and Management. 25(3), 577-601.

Epple, D. y Romano, R. (1998). Competition between private and public schools, vouchers and peergroup effects. American Economic Review, 88(1), 33-62.

Friedman, M. (1955). The role of government in education. En E. Robert (Ed.). Economics and the Public Interest (pp. 123-44). New Brunswick, New Jersey: Rutgers University Press.

Galab S., Vennam U., Komanduri A., Benny L. y Georgiadis A. (2013). The impact of parental aspirations on private school enrolment: Evidence from Andhra Pradesh, India. London: Young lives working paper, University of Oxford.

Gallego, F. (2006). Voucher-School Competition, Incentives, and Outcomes: Evidence from Chile. Job Market Paper.

Gallego, F., y Hernando, A. (2009). School choice in Chile: Looking at the Demand Side. Documento de Trabajo Instituto Economía UC, 356, 1-46.

García, C. y Paredes, R. (2010). Reducing the educational gap: Good results in vulnerable groups. Journal of Development Studies, 46(3), 535-555.

Glick, J. y Hohmann-Marriott, B. (2007). Academic performance of young children in immigrant families: The significance of race, ethnicity, and national origins. International Migration Review, 41(2), 371-402.

Hastings, J., Kane, T. y Staiger, D. (2005). Parental preferences and school competition: Evidence from a public school choice program (Working paper 11805). Cambridge: National Bureau of Economic Research.

Hastings, J. y Weinstein, J. (2007). No child left behind: Estimating the impact on choices and student outcomes (Paper Series, No. 13009). Cambridge: National Bureau of Economic Research Working.

Hsieh, C. y Urquiola, M. (2006). The effects of generalized school choice on achievement and stratification: Evidence from Chile's voucher program. Journal of Public Economics, 90(8-9), 1477-1503. 
Kleitz, B., Weiher, G., Tedin, K. y Matland, R. (2000). Choice, charter schools and household preferences. Social Science Quarterly (University of Texas Press), 81(3), 846-854.

Lafortune, J. y Tessada, J. (2016). Migrantes latinoamericanos en Chile: Un panorama de su integración social, económica y financiera. Santiago: Banco Interamericano de Desarrollo.

Long, J. y Toma, E. (1988). The determinants of private school attendance, 1970-1980. Review of Economics \& Statistics, 70(2), 351.

Lupica, C. (2016). Migración laboral en Chile: Oportunidades y desafíos para el trabajo decente. Documento de trabajo. Organización Internacional del Trabajo.

Mavisakalyan, A. (2012). Immigration and school choice in Australia. Australian Economic Review, 45(1), 29-49.

Murray, T. (2016). Public or private? The influence of immigration on native schooling choices in the United States. Economics of Education Review, (53), 268-283.

Nishimura, M. y Yamano, T. (2013). Emerging private education in Africa: Determinants of school choice in rural Kenya. World Development, (43), 266-275.

Pamuk, A. (2004). Geography of immigrant clusters in global cities: A case study of San Francisco. International Journal of Urban and Regional Research, 28(2), 287-307.

Söderstrom M. y Uusitalo R., (2010). School choice and segregation: Evidence from an admission reform. The Scandinavian Journal of Economics, 112(1), 55-76.

Tokman, A. (2005). Diagnóstico y Propuestas para la Educación Chilena. Economía Chilena 8(1), 35-52.

Weiher, G. y Tedin, K. (2002). Does choice lead to racially distinctive schools? Charter schools and household preferences. Journal of Policy Analysis and Management, 21(1), 79-92. 
